\title{
p63 (TP73L) a key player in embryonic urogential development with significant dysregulation in human bladder exstrophy tissue
}

\author{
BONNIE J. CHING ${ }^{1}$, LARS WITTLER ${ }^{2}$, JUDITH PROSKE ${ }^{2}$, GARIMA YAGNIK $^{1}$, LIHONG $^{3}{ }^{3}$, \\ MARKUS DRAAKEN $^{4,5}$, HEIKO REUTTER ${ }^{4,6}$, JOHN P. GEARHART ${ }^{7}$, \\ MICHAEL LUDWIG $^{8 *}$ and SIMEON A. BOYADJIEV ${ }^{1,7^{*}}$
}

\author{
${ }^{1}$ Section of Genetics, Department of Pediatrics, University of California Davis, 2825 50th Street, Sacramento, CA 95817, \\ USA; ${ }^{2}$ Department of Developmental Genetics, Max-Planck-Institute for Molecular Genetics, Ihnestrasse 63-73, \\ D-14195 Berlin, Germany; ${ }^{3}$ Division of Biostatistics, Department of Public Health Sciences, University of \\ California Davis, 2825 50th Street, Sacramento, CA 95817, USA; ${ }^{4}$ Department of Human Genetics; \\ ${ }^{5}$ Department of Genomics, Life and Brain Center; ${ }^{6}$ Department of Neonatology, Children's Hospital, \\ University of Bonn, Sigmund-Freud-Str. 25, D-53105 Bonn, Germany; ${ }^{7}$ Department of Urology, \\ The James Buchanan Brady Urological Institute, Johns Hopkins University, Baltimore, \\ MD 21287, USA; ${ }^{8}$ Department of Clinical Chemistry and Clinical Pharmacology, \\ University of Bonn, Sigmund-Freud-Str. 25, D-53105 Bonn, Germany
}

Received July 8, 2010; Accepted August 16, 2010

DOI: 10.3892/ijmm_00000535

\begin{abstract}
Human bladder exstrophy-epispadias complex (BEEC) comprises a spectrum of urogenital anomalies in which part or all of the distal urinary tract fails to close. Several lines of evidence implicate genetic factors in the formation of BEEC. Among them a murine $p 63^{-/}$knockout model showed the full picture of classic exstrophy of the bladder and other urogenital defects within the BEEC spectrum. This led us to study in depth the role of $p 63$ in urogenital development in mice and investigate the implication of p63 in human BEEC. Whole mount in situ analysis in mice was carried out to investigate the ventro-caudal expression of the p63 transcript at gestational days (GD) 9.5-12.5, the equivalent of human gestational weeks 4-6 (postulated time of BEEC organogenesis in humans). In addition, $p 63$ expression analysis was performed in human blood and bladder derived samples of 15 BEEC newborns accompanied by sequencing analysis of their genomic DNA. We also conducted sequencing analysis of genomic DNA in additional 22 BEEC patients. In mouse embryos, $p 63$ expression was detected at days 9.5-12.5 in the cloacal membrane and urethral epithelium, supporting its
\end{abstract}

Correspondence to: Dr Michael Ludwig, Department of Clinical Chemistry and Pharmacology, University of Bonn, Sigmund-FreudStr. 25, D-53105 Bonn, Germany

E-mail: mludwig@uni-bonn.de

*Contributed equally

Key words: bladder exstrophy, epispadias, expression analysis, $p 63$, $T P 73 L$, whole mount in situ expression role in the morphogenesis of the external genitalia and the bladder. Tissue-specific expression of a novel and alreadyknown mRNA isoforms were established and a reproducible dysregulation of variable $p 63$ isoforms was observed in 11 of 15 patients indicating altered gene expression. However, no obvious $p 63$ gene mutations were identified in any of the patients.

Our findings strongly suggest that $p 63$ is not only involved in embryonic formation of the urogenital and ventrocaudal anatomy but is also highly dysregulated in human BEEC bladder tissue. Since p63 has been shown to self-regulate its expression through a balance of its isoforms, the dysregulation observed may contribute to the formation of BEEC.

\section{Introduction}

The bladder exstrophy-epispadias-complex (BEEC) is an anterior midline defect with variable expression that affects the infraumbilical abdominal wall including the pelvis, urinary tract, and external genitalia. It ranges from isolated epispadias (E), to classic bladder exstrophy (CBE), to its most severe form, cloacal exstrophy (CE) also known as OEIS complex (omphalocele, exstrophy of the bladder, imperforate anus, spinal defects) $(1,2)$. Several lines of evidence indicate that genetic factors are involved in the etiology of BEEC, among them, a 400-fold increase of the recurrence risk for offspring of affected individuals (3), observations of rare multiplex families (4) and much higher concordance rates (62\% vs $11 \%$ ) among monozygotic as compared to dizygotic twins (5).

The strong rationale for studying $p 63$ as a candidate gene for human BEEC is based on its coordinating function during anogenital modeling and epithelial cell differentiation in the developing female mouse urogenital tract (6), and its role during the urorectal septation process deducted from murine 
knockout models ( $p 63^{-/}$) that resemble the severe human phenotypic BEEC spectrum such as $\mathrm{CE}$ or $\mathrm{CBE}(7,8)$. All 12 p63 $3^{--}$embryos examined by Cheng et al developed bladder abnormalities (8). Of those, four embryos developed CBE with ventral bladder and abdominal wall defects (with and without membrane cover), bifid external genitalia and umbilical hernia, whereas the remaining eight embryos developed dilated bladders with both thin lamina propria and thin muscle layers. In fact, sagittal sections from E18.5 $p 63^{-/-}$mutant embryos presented a range of CBE features, e.g. ventral abdominal and bladder wall defects covered with a thin membrane, absence of pubic symphysis at the midline (i.e. separation of the pubic bones), absence of external genitalia at the midline (i.e. bifid genitalia), umbilical hernia, and ventral translocation of the anus. In the absence of p63, the ventral urothelium is neither committed nor differentiated, while the dorsal urothelium is both committed and differentiated. It has been proposed that p63 is required for the maintenance of 'stemness' of all stratified epithelia $(7,9)$, or required for the very fundamental steps of commitment of and differentiation processes in stratified epithelia (9).

The $p 63($ KET/p40/p51/p73L) gene encodes at least eight protein isoforms realized by alternative splicing and alternative initiation of transcription (Fig. 1), alternate promoter usage results either in the presence (TA) or absence $(\Delta N)$ of a classical transactivation domain. By using antibodies, discriminating between only TA and $\Delta \mathrm{N}$ forms, it has been established that $\Delta \mathrm{Np} 63$ is the predominant isoform expressed throughout the bladder with a preferential expression in the ventral bladder urothelium during early development (8). It has also been shown that $\Delta N p 63$ is required for ventral specification in zebrafish. Loss of $\Delta N p 63$ results in reduction of ventral (non-neural) ectoderm, while $\Delta \mathrm{Np} 63$ overexpression expands the ventral ectoderm (10). Elimination of $\Delta N p 63$ by morpholino oligonucleotides results in embryos lacking epidermal structures and fins $(10,11)$. The epidermal proliferation regulated by $\Delta N p 63$ is achieved by dominant negative inhibition of the transcription of p53 target genes in vivo (11).

Furthermore, and contrary to the p63 murine knockout model, several human syndromes with urogenital malformations and various cleft and midline defects [limb mammary syndrome, LMS, OMIM 603543; acro-dermatoungual-lacrimal-tooth (ADULT) syndrome, OMIM 103285; ectrodactyly-ectodermal dysplasia-clefting syndrome, EECS, OMIM 604292; Hay-Wells or ankyloblepharon-ectodermal defects-cleft lip/palate (AEC) syndrome, OMIM 106260; Rapp-Hodgkin syndrome, RHS, OMIM 129400; split hand/ foot malformation type 4, SHFM4, OMIM 605289] are associated with heterozygous (dominant) p63 mutations (12).

Altogether, these findings implicate a role for $p 63$ in the etiology of human BEEC. In our study, we used whole mount in situ analysis in mice to investigate ventrocaudal expression of the p63 transcript at gestational days 9.5-11.5 in mice. This period is considered the equivalent of human gestational weeks 4-6 (13), the postulated time of BEEC development in humans (14). We also compared $p 63$ expression in cDNA samples derived from bladder tissues and lymphocytes of 15 BEEC newborns against control samples by semiquantitative PCR and quantitative real-time PCR. Finally we

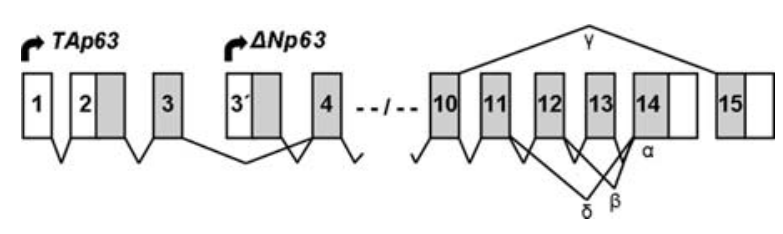

Figure 1. Schematic structure of human $p 63$. Use of different promoters (arrows) yields transactivating (TAp63) and non-transactivating ( $\Delta N p 63)$ isotypes. Alternative splicing events give rise to four mRNAs $(\alpha, \beta, \gamma, \delta)$ for both these isotypes. mRNA-type $\delta$ was identified in this study.

performed mutational analysis of genomic DNA of the entire coding region of $p 63$ among the 15 BEEC newborns as well as among an additional 22 BEEC cases.

\section{Materials and methods}

In situ hybridization of mouse embryos. Mouse embryos were dissected in ice cold PBS, fixed overnight in $4 \%$ paraformaldehyde/PBS and then processed for in situ hybridization as described (21). Digoxigenin (DIG)-labelled antisense RNA probes were transcribed in vitro from PCR products of murine p63 (nucleotide 3910-4587 of GenBank acc. no. NM_011641.1). The reverse primer contained a T7 promoter site facilitating generation of antisense probes by using the PCR product as a template. T7 RNA polymerase, transcription buffer, and nucleotide mix (Promega, Roche, Switzerland) were used according to the manufacturer's recommendations. Probes were purified using G-50 sephadex columns (GE-Healthcare, Chalfont St. Giles, UK). Photographs of whole embryos were taken with a Leica MZ16A (Wetzlar, Germany) dissecting microscope and the Zeiss Axiovision (Jena, Germany) software.

Histological analyses of mouse embryos. For the histological analyses, in situ hybridized whole mount mouse embryos were embedded in $20 \%$ albumin $/ 13 \%$ sucrose $/ 0.5 \%$ gelatine $/ 2.5 \%$ glutaraldehyde in PBS matrix. Vibratome sections with a thickness of $20 \mu \mathrm{m}$ were prepared.

Patients. The study was approved by the respective Ethics Committees of the respective institutes and informed consent was obtained from all patients and donors of normal (control) samples. Tissue and blood specimens included in the expression studies were all derived from sporadic cases ( 8 males and 7 females) with the majority of patients (15) being of North American Caucasian origin (Table I). Other anomalies of interest in these probands include ventricular septal defect in BS5 and LS5; spina bifida in LS8; renal anomalies in BS12. Patient LS1 was shown to carry a chromosomal translocation, $46, X Y, t(8 ; 9)$ (p11.2;q13) (15). Patients solely studied by $p 63$ sequence analysis were all of Central European origin, except for one patient from Morocco (70-501) and one from Panama (314-501), both recently described in detail $(16,17)$. Altogether, samples from 16 males (15 with CBE, 1 with CE) and 6 females ( 3 with $\mathrm{CBE}, 3$ with $\mathrm{CE}$ ) were investigated. Additional features associated with BEEC were observed in two of these patients, thereby implicating a BEEC-like/p63-dependent phenotype, 314-501 presented with bilateral cleft lip and 


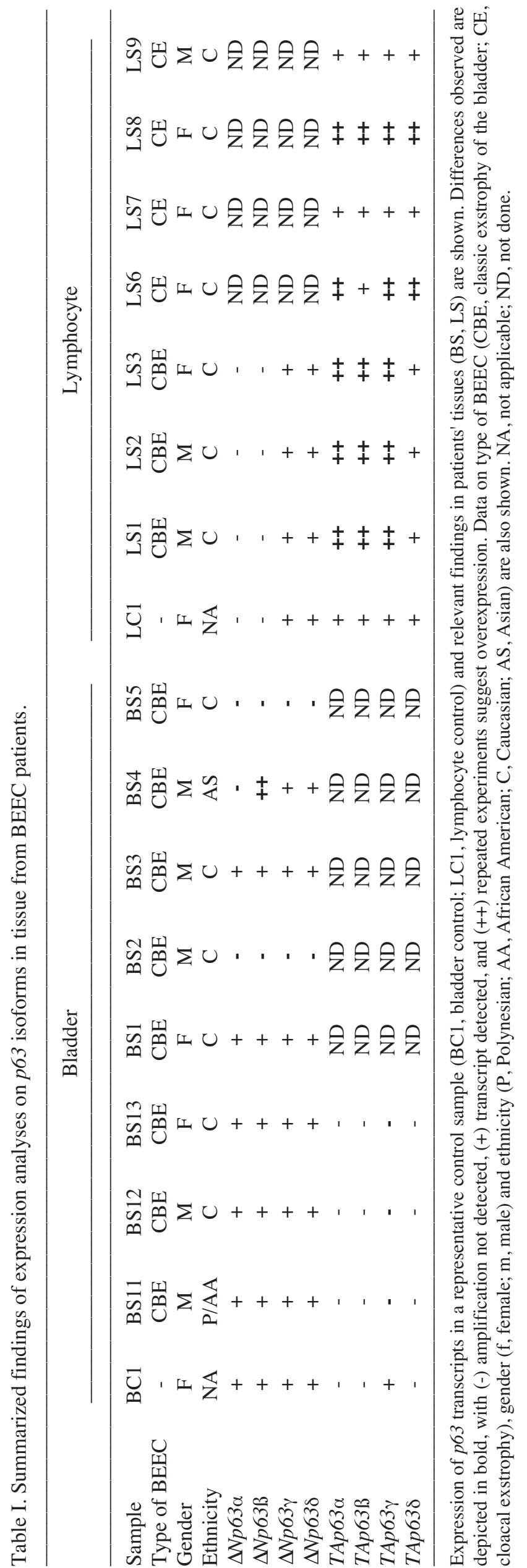

palate and patient 55-501 with bifid uvula. History of pregnancy was uneventful in all cases, without any maternal exposure to environmental toxins or maternal infections $(15,18)$.

Tissue specimens. Tissue samples from CBE/CE patients and appropriate controls were obtained at the time of surgical reconstruction during newborn period, immediately placed in RNAlater $^{\circledR}$ tissue collection, RNA stabilization solution (Ambion, Austin, TX) and stored at $-80^{\circ} \mathrm{C}$ until RNA isolation. Epstein-Barr transformed human lymphocyte cells were grown with Gibco RPMI Medium $1640+10 \%$ FBS $+1 \%$ antibiotic in $37^{\circ} \mathrm{C}+5 \% \mathrm{CO}_{2}$.

RNA and DNA analysis. Genomic DNA was isolated by standard procedures and total RNA was extracted from 50$75 \mathrm{mg}$ tissue homogenized using PolyTron or from $\sim 2 \times 10^{6}$ cells with a standard Trizol (Invitrogen, Paisley, UK) method and the RNeasy Micro Kit (Qiagen, Hilden, Germany). Integrity of the RNA was confirmed by GeneQuant pro RNA/ DNA calculator. Reverse transcription (RT) was performed using $2 \mu \mathrm{g}$ total RNA with the SuperScript First-Strand Synthesis System and for RT-PCR cDNAs derived from bladder or lymphocyte RNA were used according to the manufacturer's specifications (Invitrogen).

For sequence analysis, PCR amplification of all 16 human p63 coding exons as well as the 5' and 3'UTR regions was carried out on DNA or cDNA samples of 37 BEEC patients. Initially, in 15 patients the entire $p 63$ cDNA derived from lymphocytes or bladder RNA was sequenced (performed by UC Davis DNA Sequencing Facility). The cDNA samples showing aberrant $p 63$ expression were subsequently analyzed by genomic DNA sequencing and a detailed promoter analysis was also performed. The TAp63 promoter sequence analysis is contiguous with the 5'UTR and exon 1 (nucleotide 95,843,101-95,844,456 in acc.no. NT_005612.15) (19). The $\Delta N p 63$ promoter has also been characterized and this sequence corresponds to nucleotides 96,001,014-96,002,635 (20). In addition, solely genomic DNA was analyzed in 22 patients in order to screen for single mutations.

Expression analysis. p63 TA or $\Delta \mathrm{N}$ expression was investigated with a specific forward primer (located in either exon 1 or 3 ) combined with isoform-specific reverse primers. Carboxyterminal short products $(\sim 300 \mathrm{bp})$ were obtained with a reverse primer either directed to exon 14 or 15 and a transcript-specific forward primer.

Since short PCR amplicons ( $<200 \mathrm{bp})$ are a prerequisite for efficient real-time PCR (qPCR), we did not amplify each long isoform individually for quantitative analysis. Instead, short $T A(110 \mathrm{bp})$ and $\Delta N p 63(108 \mathrm{bp})$-specific products were amplified. These amplicons captured all four isoforms $(\alpha, \beta, \gamma$, and $\delta$ ) of the $T A$ or $\Delta N p 63$ transcript, respectively. Moreover, specific qPCR was only performed on the Nterminal of $p 63$ to observe $T A$ or $\Delta N$ family-specific expression since analysis of each individual $\mathrm{COOH}$-terminal isoform amplifies both $T A$ and $\triangle N p 63$ species. All qPCR assays consisted of 2X SYBR Green PCR Master Mix, $600 \mathrm{nM}$ of each primer, and the same amount of starting DNA $(2 \mu \mathrm{g}$ RNA synthesized into cDNA). PCR was performed on the ABI 

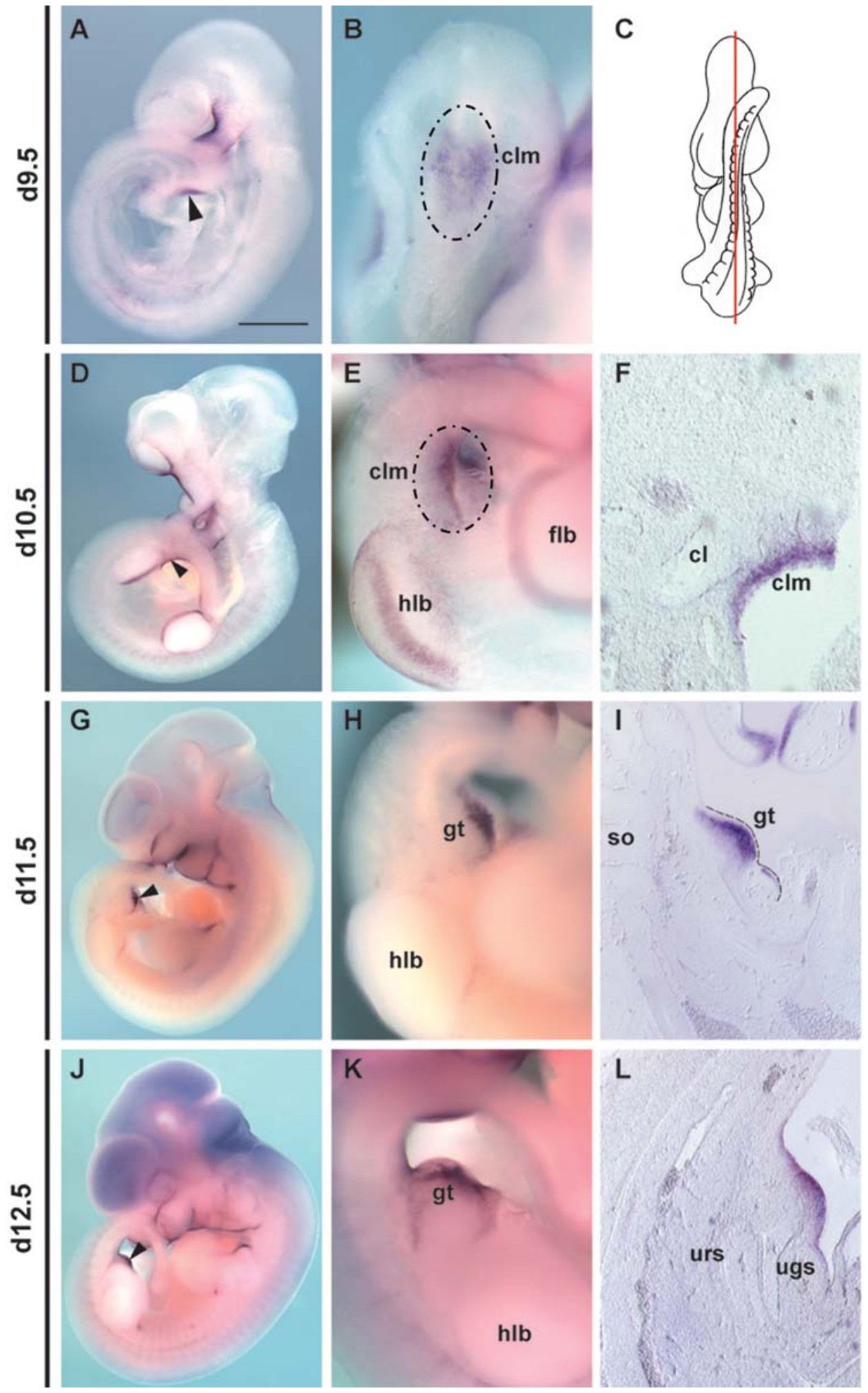

Figure 2. Analysis of $p 63$ gene expression in mouse embryos on gestation days (GDs) 9.5-12.5 using whole mount in situ hybridization. (A) Whole mount of a GD 9.5 mouse embryo. Gene expression is detectable in the ventrolateral region at the level of the cloaca (arrowhead) and in the ectoderm of the first branchial arch. (B) Close-up of the cloacal region (dashed circle) of the embryo depicted in A. (C) Schematic frontal view of a GD 9.5 embryo. The red line indicates the section plane for F, I, L. (D) Whole mount of a GD 10.5 mouse embryo. p63 expression is detectable in the forelimb and hindlimb bud, in the first and second branchial arch and in the cloacal region (arrowhead). (E) Close-up of the caudal area of the embryo circled in D. The ventral view shows a $p 63$ expression in the cloacal membrane (dashed circle) and in the apical ectodermal ridge (AER) of the forelimb and hindlimb bud. (F) Sagittal section of the caudal area of the embryo shows the presence of $p 63$ message in the ectodermal and endodermal part of the cloaca membrane. (G) Whole mount of a GD 11.5 mouse embryo where $p 63$ expression is detected in the genital tubercle (arrowhead). (H) Close-up of the ventrocaudal area of the embryo in G. This view shows the expression of $p 63$ above the genital tubercle. (I) Sagittal section of the urogenital region of the embryo in G. The genital tubercle is marked by a dashed line. Expression is detected in the cloacal membrane. (J) Whole mount of a GD 12.5 mouse embryo. p63 expression is visible in the maxillary and mandibulary arches and above the genital tubercle (arrowhead). (K) Close-up of the genital tubercle of the embryo shown in J, p63 transcripts are detected in the ectodermal compartment of the cloacal membrane. (L) Sagittal section of the urogenital region of the embryo depicted in J, showing $p 63$ expression at the genital tubercle and in the cloacal membrane. The scale bar corresponds to $165 \mu \mathrm{m}$ in A, D, G and J, $69 \mu \mathrm{m}$ in B, $330 \mu \mathrm{m}$ in C, $82 \mu \mathrm{m}$ in E, $430 \mu \mathrm{m}$ in F and I, $220 \mu \mathrm{m}$ in $\mathrm{H}$ and $110 \mu \mathrm{m}$ in K; cl, cloaca; clm, cloacal membrane, gt, genital tubercle; flb, forelimb bud; hlb, hindlimb bud; so, somites; ugs, urogenital sinus; urs, urorectal septum. 


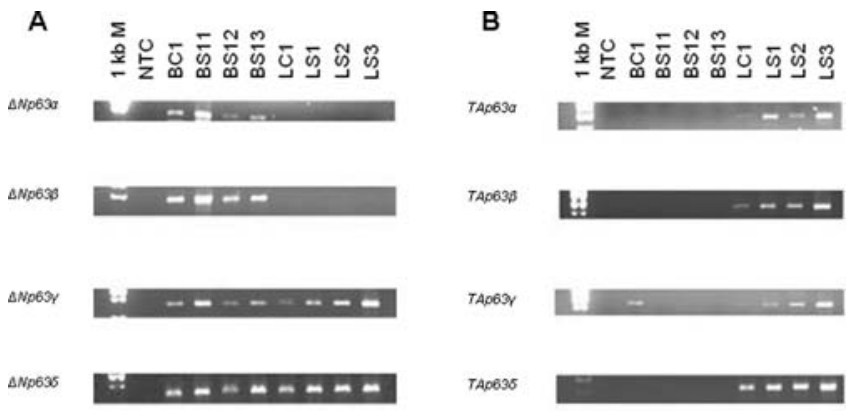

Figure 3. RT-PCR analysis of p63 NH2-terminal isoforms in bladder (BS11-13) or lymphocytes (LS1-3) obtained from control (BC1 and LC1) and CBE patients. NTC denotes no template control.

Prism 7900HT (Applied Biosystems, Foster City, USA) and each experiment was performed at least three times on separate days using the same protocol. The fold change presented for each patient was derived from the average of nine values for each amplicon. Data from all respective nine reactions were highly reproducible and there was no significant deviation between experiments. In order to precisely quantify the expression level of the individual isoform family, data were normalized in comparison to 'housekeeping' genes glyceraldehyde 3-phosphate dehydrogenase $(G A P D H)$ and $B$-actin $(A C T B)$. All primer sequences and protocols can be obtained on request.

\section{Results}

To obtain a comprehensive overview of the transcriptional activity of p63 during the development of the urogenital system and external genitalia, we performed whole mount in situ hybridization (WISH) on mouse embryos at gestational days 9.5-12.5 with particular emphasis on the region of the cloaca and the genital tubercle (Fig. 2). At day 9.5, p63 transcripts were detected in the ectodermal compartment of the cloacal membrane, as well as in the ectodermal surface of the first branchial arch and in the region of the emerging forelimb bud (Fig. 2A and 2B). At day 10.5 the expression domains in the cloacal region and in the branchial arch ectoderm persisted and the expression in the limb buds became confined to the apical ectodermal ridge (AER; Fig. 2D-F). Between day 11.5 and 12.5, during the emergence of the genital tubercle, the cloacal p63 expression became more pronounced, resulting in a strong signal in the uretral ectoderm (Fig. 2G-L).

The distribution of $p 63$ isoforms in human bladder tissue (BC1) and lymphocytic (LC1) control samples (Fig. 3B) revealed a tissue-specific expression in that all $T A$-transcripts were present in lymphocytes but TAp63 $\gamma$ was solely found in bladder tissue. Contrary, $\Delta N$-variants were all detected in bladder tissue, whereas lymphocytes only expressed the $\gamma$-type (Fig. 3A; data summarized in Table I). In the course of these studies, a novel splice variant was identified, for which the term $p 63 \delta$ (Figs. 1 and 3) was coined. Primers directed to its exon $11 / 14$ junction allowed us to confirm this yet undescribed isoform. The $\Delta \mathrm{N}-\delta$-transcript was found to be present in both bladder and lymphocyte control samples, whereas the TAp638transcript was absent in bladder controls (Fig. 3).
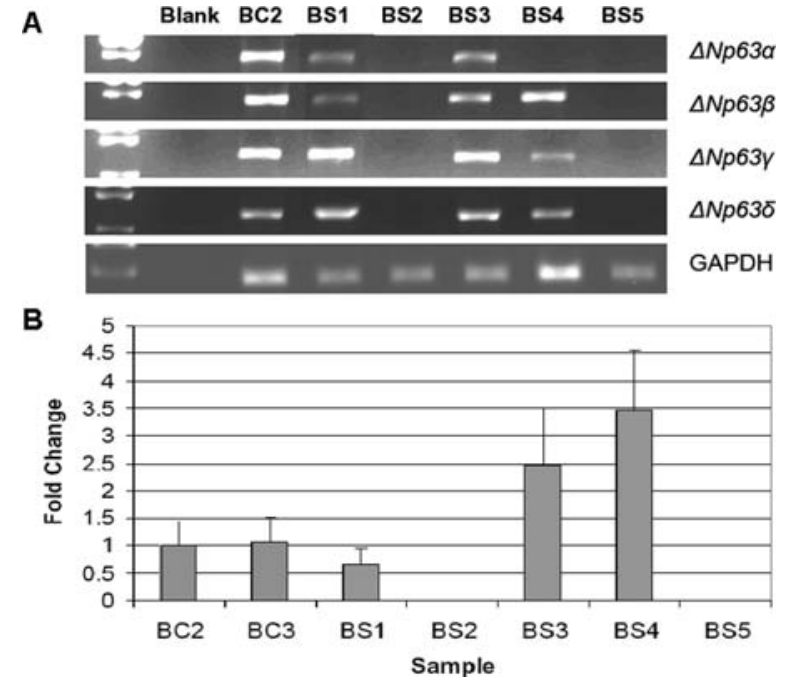

Figure 4. A. RT-PCRs of the $\Delta$ Np63 family of mRNA isoforms in different CBE bladder samples (BS1-5) compared with bladder control samples (BC2). GAPDH was used as a loading control. BS2 and BS5 lack all $\Delta N p 63$ variants and BS4 shows absence of $\Delta N p 63 \alpha$ and possible overexpression of $\Delta N p 63 \beta$. B. Cumulative qPCR of $\Delta N p 63$ family of isoforms with bladder controls (BC2-BC3) and bladder samples (S1-S5) from CBE patients. Data confirmed absence of all isoforms in BS2 and BS5 and possible overexpression of (at least) $\Delta N p 63 \beta$.
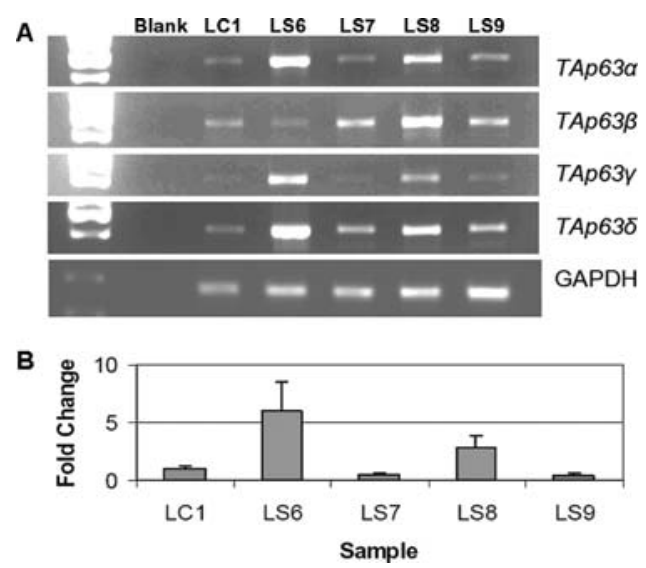

Figure 5. A. RT-PCRs of the TAp63 family of mRNA isoforms in different CE lymphocyte samples (LS6-9) compared with a control sample (LC1). $G A P D H$ was used as a loading control. Semi-quantitative PCR suggests possible overexpression of TAp63a, $-\gamma$, and $-\delta$ in LS6 and of all TAp63 isoforms in LS8. B. Cumulative TAp63 qPCR corroborates TAp63 overexpression in both, LS6 and LS8.

As seen in Fig. 3, investigation of CBE patient's samples (LS1-LS3) revealed a normal expression pattern in lymphocytes. However, bladder tissue from three CBE patients

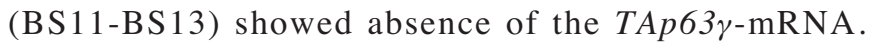
Investigation of the expressed $\Delta N$ transcripts in CBE bladder tissues detected significant alterations in another three samples (Fig. 4A). Here, BS2 and BS5 lacked all $\Delta N p 63$ variants and BS4 showed absence of $\Delta N p 63 \alpha$ with a possible overexpression of $\Delta N p 63 \beta$. These findings were verified by cumulative qPCR of all $\Delta N p 63$ isoforms (Fig. $4 \mathrm{~B}$ ).

Significant variations detected in samples from unrelated BEEC patient lymphocytes are summarized in Table I and examples are shown in Fig. 5. Semi-quantitative RT-PCR 


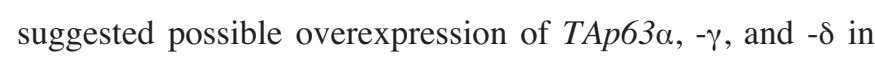
LS6 and of all TAp63 isoforms in LS8. Cumulative TAp63 real-time PCR corroborates TAp63 overexpression in both LS6 and LS8. Unfortunately, due to a very limited amount of bladder exstrophy tissue, we were unable to perform the complete series of tests with samples BS1-5 and LS6-9.

Sequencing of all $p 63$ exons and their corresponding splice junctions, promoter regions and polyA-sites revealed only four heterozygous deviations from normal in all samples tested. A silent variant (c.249T>C, D83) also detected in the unaffected father was found in patient 140-501. DNA from lymphocytes of patient LS1 showed another synonymous substitution (c.678C > T; R226R). Two patients showed an intronic variant (IVS1, $+33 \mathrm{~A} / \mathrm{G}$ and IVS3, $+76 \mathrm{C} / \mathrm{T}$, respectively). None of these substitutions has been deposited in the single nucleotide polymorphism database (NCBI dbSNP Build 131) to date.

\section{Discussion}

Our analysis of the p63 expression pattern in midgestation phase mouse embryos clearly showed the spatiotemporal correlation of $p 63$ transcriptional activity with the critical phase of urogenital development. Starting with the formation of the cloacal membrane at day $9.5, p 63$ transcription takes place in the epithelial compartiments of the cloaca, where it persists in the uretral epithelium and the adjacent ectoderm during the formation of the external genitalia. Loss of $p 63$ in mice was shown to cause limb and craniofacial defects, multiple malformations in urogenital development, and defects in the formation of the abdominal wall, resulting in exstrophy of the bladder (8). As causes for these pleiotropic defects, failures in epithelial to mesenchymal signalling as well as an antiapoptotic role of p63 were described $(7,8)$. p63 expression in the cloacal and uretral epithelium suggests that p63 is necessary for proper function as a source of patterning or proliferation signals on the mesenchyme of the adjacent lateral plate and genital tubercle mesoderm. The loss of p63 activity in this tissue can thus lead to the observed developmental defects that are a prerequisite for bladder exstrophies as described in Cheng et al (8).

Tempo-spatial expression differences of $p 63$ have been previously observed $(22,23)$, and a tissue-specific pattern including a novel $p 63 \mathrm{mRNA}$ variant, $p 63 \delta$, was also evident from our analysis of lymphocytes and bladder mRNA obtained from normal tissue. As summarized in Table I, expression differences for selective $p 63$ isoforms were identified in 11 out of 15 tested exstrophic cDNA samples and this dysbalance may be correlated with BEEC. It is known that $\mathrm{TA}$ and $\Delta \mathrm{Np} 63$ compete for the same binding sequence (24). Therefore, a decrease in some of these isoforms can be expected to result in attenuated or lost transactivation of gene targets. Since $\Delta \mathrm{Np} 63$ is required for epithelial development and formation of stratified epithelia, the lack of all or some of these isoforms might be involved in the formation of urogenital system malformations. Most interestingly is the absence of $\Delta N p 63 \alpha$ transcripts in bladder tissue from three patients (BS2, 4 and 5). It has been shown in mice that $\Delta \mathrm{Np} 63 \alpha$ protein induces expression of the extracellular matrix component Fras1, required for maintaining the integrity of the epidermaldermal interface at the basement membrane (25). Mutations in human FRAS1 have been causally linked to classical Fraser syndrome (CFS; OMIM \#219000), an autosomal-recessive defect, also known as Cryptophthalmos-Syndactyly syndrome (26). CFS shows phenotypic overlap with BEEC in that umbilical hernia (omphalocele), microphallus in males along with cryptorchidism, vaginal atresia or bicornuate uterus in females as well as diastasis of symphysis pubis in both genders are frequently observed.

Although these data strongly suggest that $p 63$ is not only involved in embryonic formation of the urogential and ventrocaudal anatomy but is also highly dysregulated in human BEEC bladder tissue, we were unable to identify genomic mutations by Sanger sequencing in BEEC patients. Though our sample size may have been too small to detect rare causal mutational events, it is unlikely that screening of larger number of samples will identify such mutations, since we failed to detect genomic mutations even in those patients, in which $p 63$ expression was significantly dysregulated. It is therefore unlikely that genomic mutations in $p 63$ are a frequent or a direct cause of BEEC in humans. However, it cannot be excluded, that we may have missed mutations in yet unknown regulatory sequences or in non-coding regions not detectable using the method applied. Also, dysfunction of other factors, involved in the regulation of $p 63$ transcription may lead to differences in its expression. Here, $p 63$ expression has been shown to be regulated by a set of specific microRNAs (miRs) in the respective tissue context (27). Therefore, expression differences observed may also be attributable to misadjusted miR expression and/or the transcription of nonfunctional miRs interfering with the precise degradation of the respective $p 63$ transcript and its quantity required.

In summary, our study showed that $p 63$ expression is consistent with direct effects on the development of the urogenital system, in particular by acting through its proposed function in epithelial stratification, cell proliferation and control of apoptosis. While our work has implicated p63 dysregulation in human bladder tissue of BEEC patients, the genetic basis for its abnormal expression has yet to be identified. Our mutation screening study did not confirm that mutations affecting $p 63$ are a frequent cause of BEEC. It remains to be elucidated to what degree these postnatal findings reflect the situation in early embryonic development when the morphogenetic events leading to BEEC occur. Future studies should consider genes encoding proteins involved in the p63 signaling pathway as possible candidates for the development of these malformations.

\section{Acknowledgements}

We wish to thank C. Nauta, A. Hata, A, Gonzalez-del Angel and Y. Lakshmanan for contacting families and providing samples. L.W., J.P., M.D., H.R. and M.L. are members of the 'Network for Systematic Investigation of the Molecular Causes, Clinical Implications and Psychosocial Outcome of Congenital Uro-Rectal Malformations (CURE-Net)' supported by a research grant (01GM08107) from the German Federal Ministry of Education and Research (Bundesministerium für Bildung und Forschung, BMBF). S.A.B. is partially supported by Children's Miracle Network endowed chair in pediatric genetics. This project has been partially supported through a 
NIH grant R01 DE016886 from NIDCD/NIH, M01-RR00052 from NCRR/NIH and by Children's Miracle Network grant CMNSB06 to SAB.

\section{References}

1. Carey JC: Exstrophy of the cloaca and the OEIS complex: One and the same. Am J Med Genet 99: 270, 2001.

2. Gearhart JP: Exstrophy, epispadias, and other bladder anomalies. In: Campbell's Urology. Walsh PC, Retik AB Vaughan ED, Wein AJ, (eds). 8th edition. W.B. Saunders Co, Philadelphia, pp2136-2196, 2002.

3. Shapiro E, Lepor H and Jeffs RD: The inheritance of the exstrophy-epispadias complex. J Urol 132: 308-310, 1984.

4. Reutter H, Shapiro E and Gruen JR: Seven new cases of familial isolated bladder exstrophy and epispadias complex (BEEC) and review of the literature. Am J Med Genet A 120A: 215-221, 2003.

5. Reutter H, Qi L, Gearhart JP, Boemers T, Ebert AK, Rösch W, Ludwig M and Boyadjev SA: Concordance analyses of twins with bladder exstrophy-epispadias complex suggest genetic etiology. Am J Med Genet A 143A: 2751-2756, 2007.

6. Ince TA, Cviko AP, Quade BJ, Yang A, McKeon FD, Mutter GL and Crum CP: p63 coordinates anogenital modeling and epithelial cell differentiation in the developing female urogenital tract. Am J Pathol 161: 1111-1117, 2002.

7. Yang A, Schweitzer R, Sun D, Kaghad M, Walker N, Bronson RT, Tabin C, Sharpe A, Caput D, Crum C and McKeon F: p63 is essential for regenerative proliferation in limb, craniofacial and epithelial development. Nature 398: 714-718, 1999.

8. Cheng W, Jacobs WB, Zhang JJR, Moro A, Park JH, Kushida M, Qiu W, Mills AA and Kim PCW: $\Delta$ Np63 plays an anti-apoptotic role in ventral bladder development. Development 133: 4783-4792, 2006.

9. Mills AA, Zheng B, Wang XJ, Vogel H, Roop DR and Bradley A: p63 is a p53 homologue required for limb and epidermal morphogenesis. Nature 398: 708-713, 1999.

10. Bakkers J, Hild M, Kramer C, Furutani-Seiki M and Hammerschmidt M: Zebrafish DeltaNp63 is a direct target of Bmp signaling and encodes a transcriptional repressor blocking neural specification in the ventral ectoderm. Dev Cell 2: 617-627, 2002.

11. Lee $\mathrm{H}$ and Kimelman D: A dominant-negative form of p63 is required for epidermal proliferation in zebrafish. Dev Cell 2: 607-616, 2002

12. Rinne T, Brunner HG and van Bokhoven H: Spotlight on p63. p63-associated disorders. Cell Cycle 6: 262-268, 2007.

13. O'Rahilly R: Early human development and the chief sources of information on staged human embryos. Eur J Obstet Gynecol Reprod Biol 9: 273-280, 1979.

14. Wood HM, Trock BJ and Gearhart JP: In vitro fertilization and the cloacal-bladder exstrophy-epispadias complex: is there an association? J Urol 169: 1512-1515, 2003.
15. Boyadjiev SA, South ST, Radford CL, Patel A, Zhang G, Hur DJ, Thomas GH, Gearhart JP and Stetten G: A reciprocal translocation $46, X Y, t(8 ; 9)(p 11.2 ; q 13)$ in a bladder exstrophy patient disrupts CNTNAP3 and presents evidence of a pericentromeric duplication on chromosome 9. Genomics 85: 622-629, 2005.

16. Reutter H, Hoischen A, Ludwig M, Stein R, Radlwimmer B, Engels H, Wolffenbüttel K and Weber RG: Genome-wide analysis for micro-aberrations in familial exstrophy of the bladder using array-based comparative genome hybridization. BJU Int 100: 646-650, 2007

17. Yokoyama E, Del Castillo V, Ramos S and González-del Angel A: Omphalocele, bladder exstrophy, imperforate anus, spine defects complex, and bilateral cleft lip and palate in one product of a triplet pregnancy obtained by in vitro fertilization: A case report. Am J Med Genet A 143A: 1933-1935, 2007.

18. Gambhir L, Höller T, Müller M, Schott G, Detlefsen B, Vogt H, Ebert AK, Fisch M, Beaudoin S, Stein R, Boyadjiev SA, Rösch W, Utsch B, Boemers TM and Ludwig M: Epidemiological survey of 214 families with bladder exstrophy-epispadias complex. J Urol 179: 1539-1543, 2008.

19. Lantner F, Starlets D, Gore Y, Flaishon L, Yamit-Hezi A, Dikstein R, Leng L, Bucala R, Machluf Y, Oren M and Shachar I: CD74 induces TAp63 expression leading to B-cell survival. Blood 110: 4303-4311, 2007.

20. Koster MI, Kim S, Huang J, Williams T and Roop DR: TAp63alpha induces AP-2gamma as an early event in epidermal morphogenesis. Dev Biol 289: 253-261, 2006.

21. Richter U, Wittler L and Kessel M: Restricted expression domains of Ezrin in developing epithelia of the chick. Gene Expr Patterns 4: 199-204, 2004.

22. Saito K, Kawakami S, Okada Y, Takazawa R, Koga F, Kageyama Y and Kihara K: Spatial and isoform specific p63 expression in the male human urogenital tract. J Urol 176: 2268-2273, 2006.

23. King KE and Weinberg WC: p63: defining roles in morphogenesis, homeostasis, and neoplasia of the epidermis. Mol Carcinog 46: 716-724, 2007.

24. Yang A, Kaghad M, Caput D, McKeon F: On the shoulders of giants: p63, p73 and the rise of p53. Trends Genet 18: 90-95, 2002.

25. Koster MI, Dai D, Marinari B, Sano Y, Costanzo A, Karin M and Roop DR: p63 induces key target genes required for epidermal morphogenesis. Proc Natl Acad Sci USA 104: 3255-3260, 2007.

26. McGregor L, Makela V, Darling SM, Vrontou S, Chalepakis G, Roberts C, Smart N, Rutland P, Prescott N, Hopkins J, Bentley E, Shaw A, Roberts E, Mueller R, Jadeja S, Philip N, Nelson J, Francannet C, Perez-Aytes A, Megarbane A, Kerr B, Wainwright B, Woolf AS, Winter RM and Scambler PJ: Fraser syndrome and mouse blebbed phenotype caused by mutations in FRAS1/Fras1 encoding a putative extracellular matrix protein. Nat Genet 34: 203-208, 2003.

27. Blandino G and Moll UM: p63 regulation by microRNAs. Cell Cycle 8: 1466-1467, 2009. 